\title{
Multimodality imaging of a hairpin-like coronary fistula between the right coronary artery and the coronary sinus
}

\author{
Yuan Peng, ${ }^{1,2}$, Yali Yang, ${ }^{1,2}$, Yuman $\mathrm{Li}^{1,2}$, Ziming Zhang ${ }^{1,2}$, Lin $\mathrm{He}^{1,2}$, \\ Lingyun Fang ${ }^{1,2}$, Chao Guo ${ }^{3}$, Nianguo Dong ${ }^{3}$, Mingxing Xie ${ }^{1,2}$ \\ ${ }^{1}$ Department of Ultrasound, Union Hospital, Tongji Medical College, \\ Huazhong University of Science and Technology, Wuhan, China \\ ${ }^{2}$ Hubei Province Key Laboratory of Molecular Imaging, Wuhan, China \\ ${ }^{3}$ Department of Cardiovascular Surgery, Union Hospital, Tongji Medical College, \\ Huazhong University of Science and Technology, Wuhan, China
}

A 37-year-old man was referred to our hospital with a 2-year history of chest pain. It frequently occurred during moderate exercise. Coronary computed tomography angiography (CTA) showed a dilated tortuous right coronary artery (RCA) (Fig. $1 \mathrm{~A}-\mathrm{C})$. His clinician suspected that he might have suffered from Kawasaki disease in his childhood and arranged for an echocardiographic examination. Transthoracic echocardiography showed a dilated RCA and an enlarged coronary sinus (CS) with a bright flow (Fig. 1D). Continuous-wave Doppler revealed a continuous-wave flow at the coronary sinus (CS). Transesophageal echocardiography (TEE) demonstrated a tortuous coronary fistula from the distal RCA, which connected with the CS aneurysm through a $5 \mathrm{~mm}$ wide defect (Fig. $1 \mathrm{E}, \mathrm{F}$ ). The digital subtraction angiography confirmed the hairpin-like RCA-to-CS fistula, which was difficult to plug (Fig. 1G, Suppl. Video 1). The mean pulmonary arterial pressure was $30 \mathrm{mmHg}$, and the right ventricle was slightly enlarged. The patient was then taken for surgical treatment. The CS aneurysm was incised longitudinally adjacent to the great cardiac vein, allowing operative closure of the secluded fistula (Fig. 1H, I). The post-procedure TEE demonstrated no shunt. The patient made a rapid recovery after the operation, and had no chest pain during exercise at 3-month follow-up.

Coronary artery fistula (CAF) is a rare cardiac anomaly. CTA is recognized to be very accurate in order to diagnose CAF. The missed diagnosis resulted from the fact that this fistula came from the distal RCA and the defect was concealed in the CS. However, multi-modal imaging can provide anatomical and hemodynamic information of this rare RCA-to-CS fistula for choosing a reliable treatment.

Conflict of interest: None declared

Address for correspondence: Mingxing Xie, MD, PhD, 1277 Jiefang Avenue, Wuhan, China, tel: + 86 (27) 85726430 , fax: +86 (27) 85726386, e-mail: xiemx@hust.edu.cn; Nianguo Dong, MD, PhD, 1277 Jiefang Avenue, Wuhan, China, tel: +86 13971181551, fax: +86 (27) 85726386, e-mail: dongnianguo@hotmail.com

Received: $14.10 .2020 \quad$ Accepted: 7.12.2020

${ }^{\#}$ Both authors contributed equally to this manuscript.

This article is available in open access under Creative Common Attribution-Non-Commercial-No Derivatives 4.0 International (CC BY-NC-ND 4.0) license, allowing to download articles and share them with others as long as they credit the authors and the publisher, but without permission to change them in any way or use them commercially. 


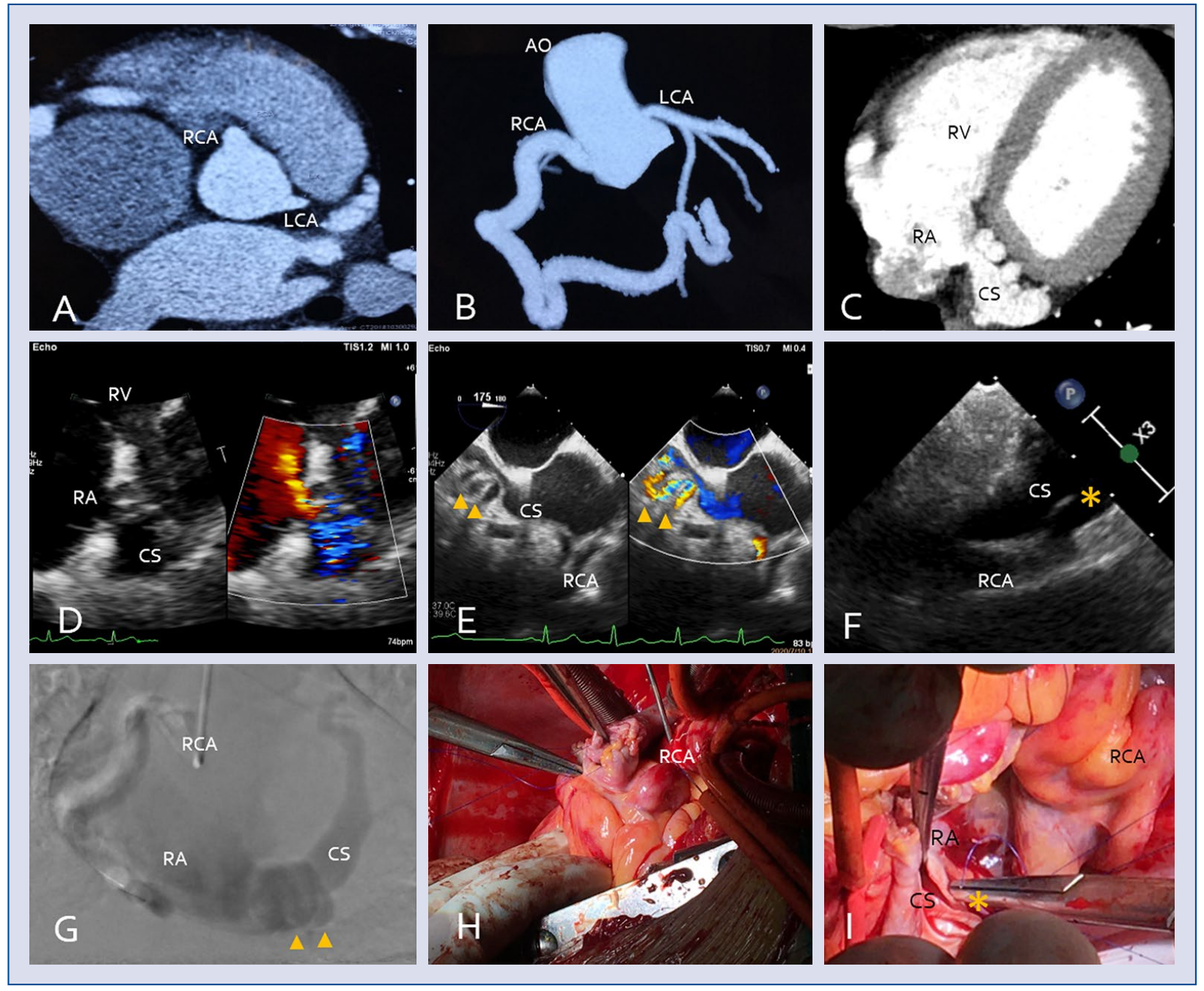

Figure 1. A-C. The coronary artery computed tomography angiography (CTA) showed the origin of a tortuous dilated right coronary artery (RCA), its course and a dilated coronary sinus (CS); D. Transthoracic echocardiography showed a bright flow from the dilated CS; E, F. Transesophageal echocardiography showed a hairpin-like coronary fistula and the defect between the distal RCA and the CS aneurysm (arrow, asterisk); G. The angiography demonstrated the termination of the RCA fistula (arrow); H, I. At surgery, the tortuous dilated RCA can be seen, while the defect was concealed in the CS (asterisk); LCA — left coronary artery; AO — aorta; RA — right atrium; RV - right ventricle. 